\section{On Reconstruction Methods for Processing Finite-Length Signals with Paraunitary Filter Banks}

\author{
Ricardo L. de Queiroz and K. R. Rao
}

\begin{abstract}
New expressions are developed for the perfect reconstruction of the boundary regions of a finite-length signal after subband processing. The time-invariant filter bank is required to be uniform and paraunitary, using FIR filters regardless of phase or symmetry. They accomodate a linear boundary extension in the analysis section, and avoid periodic extensions or storage of extended subband signals. The reconstruction methods are based on the formulation of linear systems that are built as a function of the filters.
\end{abstract}

\section{INTRODUCTION}

The theory of multirate filter banks [1]-[3] often assumes infinite length signals, while the problem of processing a finite length signal has recently attracted the attention of some researchers [4]-[13]. Finite-length 1-D signals are frequently used to model images assuming separable transforms. Recently, they were also studied to construct time-varying wavelet packets [14], [15]. The study of the boundary distortion is nearly as old as the idea of subband coding of images, including periodic extensions and the use of convolution in DFT domain [4], inclusion of few extra samples [5], or perhaps a simple study of which extension would minimize the border distortions [6]. However, it is quite easy to see that if the extension and the subband filters are symmetric, the deleted subband samples could be recovered by a simple symmetric extension of the subband signal [7]-[10]. In general, two-channel filter banks are assumed. In [10] and [11], these results were extended for more than two channels, and in [11] a reconstruction method was developed for nonlinear phase filters. In [12], an alternative approach to [11] was proposed. Size-limited filter banks are discussed in [13] and there are several proposals based on applying special filter banks (basis functions) to the borders in order to assure full orthogonality [14], [15]

Assume the finite-length signal $r(n)$ has $N_{s}$ samples and let $\mathbf{x}=\left[x(0), \cdots, x\left(N_{S}-1\right)\right]^{T}$. A nonexpansive analysis system will convert $\mathbf{x}$ into $N_{S}$ subband samples, which we similarly merge into vector $\mathbf{y}$, for simplicity. After processing or quantization, the resulting subband vector $\hat{\mathbf{y}}$ is submitted to a synthesis system that will recover the vector $\hat{\mathbf{x}}$. Following [13], models for the size-limited analysis or synthesis systems are shown in Fig. 1(a) and Fig. 1(b), respectively. In these models, finite-length processing is accomplished by converting the signal to a symmetric-periodic sequence, which is processed and windowed. On the other hand, for real filter banks there is a linear transform $\mathbf{G}$ such that $\mathbf{y}=\mathbf{G x}$. Thus, the perfect reconstruction synthesis is accomplished by $\hat{\mathbf{x}}=\mathbf{G}^{-1} \hat{\mathbf{y}}$. However, it is not always practical to invert a $N_{S} \times N_{S}$ matrix, nor to perform analysis or synthesis through a $N_{S} \times N_{S}$ linear transform. We will deal here with uniform paraunitary FIR filter banks, with arbitrary phase response. The popular linear-phase filters are a particular example, and although the results here can be surely applied in such

Manuscript received September 8, 1993; revised August 18, 1994. This work was supported in part by the Conselho Nacional de Desenvolvimento Científico e Tecnológico (CNPq), Brazil, under Grant 200.804-90-1. The associate editor coordinating the review of this paper and approving it for publication was Prof. Roberto H. Bamberger

The authors are with the Electrical Engineering Department, University of Texas at Arlington, Arlington, TX 76019 USA.

IEEE Log Number 9413849.

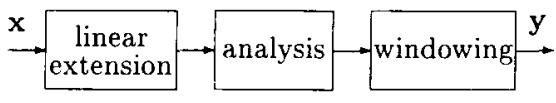

(a)

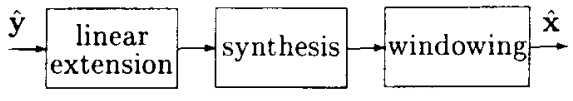

(b)

Fig. 1. Extension and windowing in the analysis and synthesis of a finite-length signal. The finite-length signal is transformed into an infinite-length signal by assuming a linear (e.g. periodic) extension. After processing, the desired sequence is extracted from the resulting signal by applying a rectangular window. (a) Overall analysis section. (b) Overall synthesis section.

a case, they may offer a simpler solution because of the assumed generality of the impulse responses of the filters. In addition, we do not impose any symmetry restriction for the boundary extension process. Under these conditions, given the boundary extension used in the analysis section and the filter bank, we prove in the appendix that assuming a linear boundary extension, $\mathbf{G}$ is always one-to-one and onto, therefore, its inverse is unique. Also, all reconstruction methods lead to identical results and have the same sensitivity to quantization or processing of the subbands. We will limit ourselves to the case where only time or subband samples are used to reconstruct the original signal (while a combination of both can be found in [11] and [12]) and assume $\hat{\mathbf{y}}=\mathbf{y}$.

In terms of notation, our conventions are: unidimensional concatenation of matrices and vectors is indicated by a comma; []$^{T}$ means transposition, while []$^{+}$and []$^{-}$stand for pseudo-inverses of a matrix, as $\mathbf{A}^{+}=\left(\mathbf{A}^{T} \mathbf{A}\right)^{-1} \mathbf{A}^{T}$ and $\mathbf{A}^{-}=\mathbf{A}^{T}\left(\mathbf{A} \mathbf{A}^{T}\right)^{-1} ; \mathbf{I}_{n}$ is the $n \times n$ identity matrix; $\mathbf{0}_{n}$ is the $n \times n$ null matrix; and $\mathbf{J}_{n}$ is the $n \times n$ counter-identity, reversing, or exchange matrix. For example

$$
\mathbf{J}_{4}=\left[\begin{array}{llll}
0 & 0 & 0 & 1 \\
0 & 0 & 1 & 0 \\
0 & 1 & 0 & 0 \\
1 & 0 & 0 & 0
\end{array}\right] .
$$

\section{Transform MatriX of a Paraunitary Filter Bank}

We assume an FIR uniform paraunitary filter bank (PUFB). We have $M$ analysis and synthesis filters. Let $L$ be the maximmum number of taps and we define $L$ to be a multiple of $M$, as $L=N M$, where $N$ is the smaller integer such that the maximum filter length lies between $N M$ and $(N-1) M$. If a filter's impulse response does not have length $L$, we pad zeros so as to reach length $L$. The analysis and synthesis filters are, thus, denoted as $f_{k}(n)$ and $g_{k}(n)$, respectively, $(k=0.1, \cdots, M-1: n=0,1, \cdots, L-1)$. Let the input signal $x(n)$ have its polyphase components denoted as $x_{i}(m)=x(m M+i)$ and the subband signals be denoted as $y_{i}(m) .(i=0.1, \cdots, M-1: m$ integer.) Define a signal $y(n)$ whose polyphase components are the subband signals, i.e., $y_{i}(m)=$ $y(m M+i)$. It is well known that a PUFB is a special form of a block filter [1], governed by a paraunitary FIR transfer matrix $\mathbf{E}(z)$, which relates the polyphase components of $x(n)$ and $y(n)$. Also, $\mathbf{E}(z)$ has $N$ impulse response matrices [1]. For a PUFB [2], [3] a transform matrix $\mathbf{P}$ of size $M \times L$ and elements $p_{i j}(i=0.1, \cdots, M-1$ : $j=0,1, \cdots, L-1$ ) can be defined as

$$
p_{i j}=f_{i}(L-1-j)=g_{i}(j) .
$$


$\mathbf{P}$ can be divided into $N$ square sub-matrices, yielding

$$
\mathbf{P}=\left[\mathbf{P}_{0}, \mathbf{P}_{1}, \cdots, \mathbf{P}_{N-1}\right] \text {. }
$$

Therefore, we can express $\mathbf{P}$ as the impulse response matrices of $\mathbf{E}(z)$ as

$$
\mathbf{E}(z)=\sum_{l=0}^{N-1}\left(\mathbf{P}_{N-1-l} \mathbf{J}_{M l}\right) z^{-l} .
$$

The paraunitariness of $\mathbf{E}(z)$, i.e., $\mathbf{E}(z) \mathbf{E}^{T}\left(z^{-1}\right)=\mathbf{E}^{T}\left(z^{-1}\right) \mathbf{E}(z)=$ $\mathbf{I}_{M}$, is also given by [2]

$$
\sum_{m=0}^{N-1-l} \mathbf{P}_{m} \mathbf{P}_{m+l}^{T}=\sum_{m=0}^{N-1-l} \mathbf{P}_{m+l} \mathbf{P}_{m}^{T}=\delta(l) \mathbf{I}_{M}
$$

where $\delta(l)$ is the Kronecker delta. We will use the matrix notation for the analysis and synthesis using PUFB's that basically follow a noncausal notation (see [1] for details on paraunitary systems and filter banks and [2] and [3] for details in using transform matrices in the description of PUFB's). Let $\mathbf{x}$ and $\mathbf{y}$ represent the timedomain and subband vectors of $N_{S}$ samples (containing signals $x(n)$ and $y(n)$ ). Using linear boundary extensions, $\mathbf{x}$ is first extended by $\lambda=(L-M) / 2$ samples in each border, originating the vector $\dot{\mathbf{x}}$, which is, thus, transformed by matrix $\mathbf{T}$, resulting in the subband vector $\mathrm{y}$. Assume the signal has $N_{S}=N_{B} M$ samples, where $N_{B}$ is an integer, then $\mathbf{T}$ is a block-Toeplitz-like matrix [2], [3] given by

$$
\mathbf{T}=\left(\begin{array}{cccccccc}
\ddots & & & \ddots & & & & 0 \\
& \mathbf{P}_{0} & \mathbf{P}_{1} & \cdots & \mathbf{P}_{N-1} & & & \\
& & \mathbf{P}_{0} & \mathbf{P}_{1} & \cdots & \mathbf{P}_{N-1} & & \\
& & & \mathbf{P}_{0} & \mathbf{P}_{1} & \cdots & \mathbf{P}_{N-1} & \\
0 & & & & \ddots & & & \ddots
\end{array}\right) .
$$

Note that $\mathbf{T}$ has $N_{B}$ block-rows, is a nonsquare matrix $\left(N_{S} \times N_{S}+\right.$ $2 \lambda$ ), and it cannot be inverted. Denoting by $\hat{\mathbf{x}}$ and $\hat{\mathbf{y}}$ the vectors in the synthesis section, analysis and synthesis are described by [2], [3]

$$
\mathbf{y}=\mathbf{T} \tilde{\mathbf{x}} \quad \hat{\tilde{\mathbf{x}}}=\mathbf{T}^{T} \hat{\mathbf{y}} .
$$

The PR conditions in (4) tell us that $\mathbf{T} \mathbf{T}^{T}=\mathbf{I}_{N_{S}}$, but, obviously, $\mathbf{T}^{T} \mathbf{T} \neq \mathbf{I}$. If $\hat{\mathbf{y}}=\mathbf{y}$ (no processing or quantization), we have

$$
\hat{\tilde{\mathbf{x}}}=\mathbf{T}^{T} \mathbf{T} \tilde{\mathbf{x}} \text {. }
$$

\section{Reconstructing Distorted DATA}

The analysis operation would require the knowledge of $\lambda$ samples outside the range of $\mathbf{x}$. Setting these samples to zero or using circular convolution can generate undesirable high-frequency components due to discontinuities at the borders. The extension of $\mathbf{x}$ into $\tilde{x}$ will be restricted here to be a linear boundary extension where the unknown samples are found by a linear combination of samples contained in $\mathbf{x}$. We assume that, for each border, the $\lambda$ samples accross the border are found as a linear function of at most $\lambda$ boundary samples of the signal. We adopt the notation shown Fig. $2^{l}$ where $\mathbf{x}$ is divided into three regions as $\mathbf{x}^{T}=\left[\mathbf{x}_{l}^{T}, \mathbf{x}_{c}^{T}, \mathbf{x}_{r}^{T}\right]$, and the extended vector is formed by $\tilde{\mathbf{x}}^{T}=\left[\mathbf{x}_{\epsilon, l}^{T}, \mathbf{x}^{T}, \mathbf{x}_{\epsilon, r}^{T}\right]$. Then, we have $\tilde{\mathbf{x}}^{T}=\left[\mathbf{x}_{\epsilon, l}^{T}, \mathbf{x}_{l}^{T}, \mathbf{x}_{c}^{T}, \mathbf{x}_{r}^{T}, \mathbf{x}_{\epsilon, r}^{T}\right]$, where

$$
\mathbf{x}_{t, l}=\mathbf{R}_{l} \mathbf{x}_{l} . \quad \mathbf{x}_{e, r}=\mathbf{R}_{r} \mathbf{x}_{r} .
$$

The size of each subvector is indicated in Fig. 2 and $\mathbf{R}_{l}$ and $\mathbf{R}_{r}$ are arbitrary $\lambda \times \lambda$ matrices to extend the signal on the left and right

\footnotetext{
1 The expressions left and right are used to designate the extremes of the vector $\mathbf{x}$ just as if it is displaced horizontally. In this case, $x(0)$ is the leftmost sample, while $x\left(N_{S}-1\right)$ is the rightmost one.
}
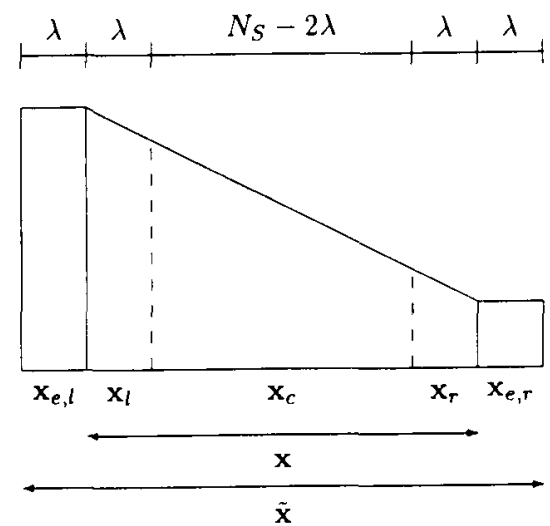

Fig. 2. Illustration of signal extension of vector $\mathbf{x}$ into vector $\tilde{\mathbf{x}}$. In each border, $\lambda=(L-M) / 2$ samples outside initial signal boundaries are found by linear relations applied to the $\lambda$ boundary samples of $\mathbf{x}$, i.e., $\mathbf{x}_{t . l}=\mathbf{R}_{l} \mathbf{x}_{l}$ and $\mathbf{x}_{t, r}=\mathbf{R}_{r} \mathbf{x}_{r}$. As only $\lambda$ samples are affected across the signal boundaries, it is not necessary to use the infinite-length extension. Also, $\mathbf{x}_{l}$ and $\mathbf{x}_{r}$ contain the samples possibly affected by the border distortions after synthesis.

borders, respectively. For example, a popular extension method is the symmetric extension [7], [10], which is mainly inherited from the use of linear-phase filters, i.e., $\mathbf{R}_{l}=\mathbf{R}_{r}=\mathbf{J}_{\lambda}$.

We will show solutions for the left border and the reader can easily infer the solution for the right border by simply reversing $\mathrm{x}$ and the columns of $\mathbf{P}$. In (7), using (4), we see that

$$
\mathbf{H}=\mathbf{T}^{T} \mathbf{T}=\left[\begin{array}{lll}
\mathbf{H}_{L} & & 0 \\
& \mathbf{I}_{\alpha_{S}-2 \lambda} & \\
\mathbf{0} & & \mathbf{H}_{R}
\end{array}\right] .
$$

The matrix $\mathbf{H}$ is block diagonal and $\mathbf{H}_{l}$ and $\mathbf{H}_{R}$ are $2 \lambda \times 2 \lambda$ matrices. For the left border, if we let

$$
\Phi=\left[\begin{array}{cccc}
\mathbf{P}_{0} & \mathbf{P}_{1} & \cdots & \mathbf{P}_{X-2} \\
& \mathbf{P}_{0} & \cdots & \mathbf{P}_{V-3} \\
& & \ddots & \\
0 & & & \mathbf{P}_{0}
\end{array}\right]
$$

then

$$
\mathbf{H}_{L}=\Phi^{T} \Phi .
$$

From (7) and (9), and dividing $\mathbf{H}_{L}$ into two equal parts as $\mathbf{H}_{L}=$ $\left[\mathbf{H}_{t, l}, \mathbf{H}_{l}\right]$, we have

$$
\left[\begin{array}{c}
\hat{\mathbf{x}}_{t, l} \\
\hat{\mathbf{x}}_{l}
\end{array}\right]=\left[\mathbf{H}_{\epsilon . l,}, \mathbf{H}_{l}\right]\left[\begin{array}{c}
\mathbf{x}_{t . l} \\
\mathbf{x}_{l}
\end{array}\right]
$$

Hence, from (8)

$$
\left[\begin{array}{c}
\hat{\mathbf{x}}_{t . l} \\
\hat{\mathbf{x}}_{l}
\end{array}\right]=\left(\mathbf{H}_{+. l} \mathbf{R}_{l}+\mathbf{H}_{l}\right) \mathbf{x}_{l}
$$

and

$$
\mathbf{x}_{l}=\left(\mathbf{H}_{t . l} \mathbf{R}_{l}+\mathbf{H}_{l}\right)^{+}\left[\begin{array}{c}
\hat{\mathbf{x}}_{t, l} \\
\hat{\mathbf{x}}_{l}
\end{array}\right] .
$$

Thus, $\mathbf{x}_{l}$ is recovered from the distorted extended signal using only linear relations.

\section{Subbands Extension to PREVEnt Distortion}

The reconstruction problem is caused by the deletion of extra subband samples (resulting from the convolution with the subband filters) by the windowing process. There are $I$ blocks of $M$ of these samples deleted from each border, where $K$ is the largest integer smaller than or equal to $N / 2$. If we could infer these samples from the 
samples actually retained along with the subbands, we could extend the subbands, use one algorithm for the whole synthesis section, and window the output without any distortion. As in the previous case, we present the solution for the left border, and the reader can infer the solution for the right border, by reversing $\mathbf{x}$, the subband signals, and the columns of $\mathbf{P}$. Let $\mathbf{x}_{\mathrm{in}}^{T}=[x(0), x(1), \cdots, x(L-$ $M-1)]$ and $\mathrm{y}_{\mathrm{in}}^{T}=[y(0), y(1), \cdots, y(K M-1)]$. Note that $\mathbf{y}_{\mathrm{i} 1}^{T}=\left[y_{0}(0), \cdots, y_{M-1}(0), y_{0}(1), \cdots, y_{M-1}(1), \cdots, y_{0}\left(K^{-}-\right.\right.$ 1), $\left.\cdots, y_{M-1}(K-1)\right]$. Let $\mathbf{E}_{T}$ and $\mathbf{E}_{\digamma}$ be square matrices and $\mathbf{x}_{\text {out }}$ and $\mathbf{y}_{\text {out }}$ be vectors, such that

$$
\mathbf{y}_{\text {out }}=\mathbf{E}_{H} \mathbf{y}_{\text {in }} \quad \text { if } \quad \mathbf{x}_{\text {out }}=\mathbf{E}_{T} \mathbf{x}_{\mathrm{in}} .
$$

The physical meaning of these equations is: if $\mathbf{x}_{\text {out }}$ is an extension vector in time domain, then the extended subband signals are contained in $\mathbf{y}_{\text {out }}$. The relation between extended samples and samples actually existing in the signal is given by the matrices for extension in frequency (subbands) and time domains, $\mathbf{E}_{k}$ and $\mathbf{E}_{T}$, respectively.

Let, also, $\mathbf{F}$ be a matrix similar to $\mathbf{T}$ but with only $2 K^{-}$blockrows, and assume $\mathbf{F}$ is divided into four $K M \times(N+2 K-1) M / 2$ submatrices.

$$
\mathbf{F}=\left(\begin{array}{ll}
\mathbf{F}_{00} & \mathbf{F}_{01} \\
\mathbf{F}_{10} & \mathbf{F}_{11}
\end{array}\right)
$$

For example, for $N=3$, we have $\mathbf{F}=\left[\begin{array}{llll}\mathbf{P}_{0} & \mathbf{P}_{1} & \mathbf{P}_{2} & \mathbf{0}_{M} \\ \mathbf{0}_{M} & \mathbf{P}_{0} & \mathbf{P}_{1} & \mathbf{P}_{2}\end{array}\right]$. We want to find a matrix $\mathbf{E}_{F}$ such that, for a given $\mathbf{E}_{T}$

$$
\left[\begin{array}{c}
\mathbf{y}_{\text {out }} \\
\mathbf{y}_{\text {in }}
\end{array}\right]=\mathbf{F}\left[\begin{array}{c}
\mathbf{x}_{\text {out }} \\
\mathbf{x}_{\text {in }}
\end{array}\right] \text {. }
$$

Thus

$$
\begin{aligned}
{\left[\begin{array}{cc}
\mathbf{E}_{f} \mathbf{y}_{\mathrm{in}} \\
\mathbf{y}_{\mathrm{in}}
\end{array}\right] } & =\left[\begin{array}{ll}
\mathbf{F}_{00} & \mathbf{F}_{01} \\
\mathbf{F}_{10} & \mathbf{F}_{11}
\end{array}\right]\left[\begin{array}{cc}
\mathbf{E}_{T} & \mathbf{x}_{\mathrm{in}} \\
\mathbf{x}_{\mathrm{in}}
\end{array}\right] \\
& =\left[\begin{array}{ll}
\mathbf{F}_{00} & \mathbf{E}_{T}+\mathbf{F}_{01} \\
\mathbf{F}_{10} & \mathbf{E}_{T}+\mathbf{F}_{11}
\end{array}\right] \mathbf{x}_{\mathrm{in}}
\end{aligned}
$$

The above equation carries two equalities. Substituting one in the other, we get

$$
\mathbf{E}_{F}\left(\mathbf{F}_{10} \mathbf{E}_{T}+\mathbf{F}_{11}\right) \mathbf{x}_{\mathrm{in}}=\left(\mathbf{F}_{00} \mathbf{E}_{T}+\mathbf{F}_{01}\right) \mathbf{x}_{\mathrm{in}} .
$$

Disregarding $\mathbf{x}_{\mathrm{in}}$ and equating the matrices, we have one solution as

$$
\mathbf{E}_{F}=\left(\mathbf{F}_{00} \mathbf{E}_{T}+\mathbf{F}_{01}\right)\left(\mathbf{F}_{10} \mathbf{E}_{T}+\mathbf{F}_{11}\right)^{-} \text {. }
$$

For the special case of linear-phase filters, $\mathbf{E}_{F}$ is simplified to a diagonal matrix with \pm 1 as the diagonal elements, according to the symmetry of the filters [11], having, thus, a trivial implementation. In this case, symmetric extensions are often applied [7]-[10].

\section{Conclusion}

We intended to show techniques to effectively achieve perfect reconstruction of the signal, regardless of the phase of the filters. Also, only a few restrictions are made on the boundary linear extensions. However, properties of these extensions are not explored here. We are also limited to uniform FIR PUFB, but the same solution can be applied to nonuniform filter banks, given that they could be constructed by cascading uniform ones. It should be noted that all the solutions using linear extension and time-invariant ${ }^{2}$ filter banks have very different approaches but are identical in essence and results. Of course, this assumes that the entire computation uses real arithmetic or suffers negligible rounding effects, compared to the effects of subband

\footnotetext{
2 The filter bank can always be viewed as time-varying due to the borders. However, the expression time-invariant here implies processing over an extended sequence resulting from linear extension of the signal.
}

quantization. So, sensitivity to quantization errors is only a function of the PUFB and of the extension method.

In order to compare our results with other results applicable to nonlinear-phase PUFB's, we may rule out the direct inversion of $G$ because of the amount of computation (for inversion and for the analysis or synthesis transformation) involved for large $\nu_{s}$, even considering $\mathbf{G}$ is a relatively sparse matrix. Both solutions in [11] and [12], as well as those presented here, allow the use of any algorithm designed for the PUFB in question. However, the solutions in [11] and [12] lead to the evaluation of systems, for each border, of the form $\mathbf{v}=\mathbf{A s}+\mathbf{B t}$, where $\mathbf{A}$ and $\mathbf{B}$ are real matrices and $\mathbf{v}$ ( $\lambda$ elements), $\mathbf{s}(2 \hbar M$ elements $)$, and $\mathbf{t}$ ( $2 \lambda$ elements) are vectors corresponding to reconstructed samples, subband samples, and time-domain samples, respectively. ([11] assumed $X$ even, while [12] assumed $N$ odd.) Here, subband and time-domain samples are not computed together, which makes the implementation easier. Furthermore, we require performing one linear transform per border, where the operator matrix can have size $\lambda \times 2 \lambda(14)$ or $K M \times K M$ (15), reflecting computational savings over [11], [12].

\section{APPENDIX}

To evaluate if $\mathbf{G}$ has indeed full-rank, regardless of the linear extension, let

$$
\mathbf{x}_{\infty}^{T}=\left[\cdots, \mathbf{x}^{T} \cdot(\mathbf{R x})^{T} \cdot \mathbf{x}^{T} \cdot(\mathbf{R x})^{T} \cdot \mathbf{x}^{T} \cdot(\mathbf{R x})^{T} \cdot \cdots\right]
$$

where $\mathbf{R}$ combines $\mathbf{R}_{i}$ and $\mathbf{R}_{r}$. In the worst case we may impose that $N_{S} \geq 2 \lambda$. The signal represented by $\mathbf{x}_{\infty}$ is periodic with period $2 N_{S}$ given by $\mathbf{x}_{\text {per }}^{T}=\left[\mathbf{x}^{T},(\mathbf{R x})^{T}\right]$. Define $\mathbf{T}_{\infty}$ as $\mathbf{T}$ but with an infinite number of block-rows. Hence, $\mathbf{y}_{x}=\mathbf{T}_{\infty} \mathbf{x}_{\infty}$ is also periodic composed by vectors of the form $\mathbf{y}_{\mathrm{per}}^{T}=\left[\mathbf{y}^{T} \cdot \mathbf{y}_{\mathrm{v}}^{T}\right]$, where nothing is said about the relation between $y$ and $\mathbf{y}_{\imath}$. There exists a linear relation between $\mathbf{y}_{\text {per }}$ and $\mathbf{x}_{\text {per }}$ such that $\mathbf{y}_{\text {per }}=\mathbf{T}_{\text {per }} \mathbf{x}_{\text {per }}$, where $\mathbf{T}_{\text {per }}$ is an orthogonal block circulant matrix [3]. Rewritting this relation, dividing $\mathbf{T}_{\text {per }}$ into four $N_{S} \times N_{S}$ submatrices, we have

$$
\left[\begin{array}{c}
\mathbf{y} \\
\mathbf{y}_{\bullet}
\end{array}\right]=\left[\begin{array}{ll}
\mathbf{T}_{00} & \mathbf{T}_{01} \\
\mathbf{T}_{10} & \mathbf{T}_{11}
\end{array}\right]\left[\begin{array}{c}
\mathbf{x} \\
\mathbf{R x}
\end{array}\right]
$$

Then

$$
\mathbf{y}=\left[\mathbf{T}_{00}+\mathbf{T}_{01} \mathbf{R}\right] \mathbf{x}=\mathbf{G} \mathbf{x}
$$

Since $\mathbf{x}$ can be any vector in $\mathrm{R}^{N_{S}}$, all possible combinations of elements of $x_{\text {per }}$ span a subspace of $R^{2 N_{S}}$ of dimension $N_{S}$. As $\mathbf{T}_{p e,}$ is orthogonal, $\operatorname{rank}\left\{\left[\mathbf{T}_{00}, \mathbf{T}_{01}\right]\right\}=V_{S}$ (full rank), and from (22), all possible combination of elements of $y$ span $x^{\nu_{s}}$. Therefore, as the same applies for $\mathbf{x}$, we see from (23) that $\mathbf{G}$ has full rank and is one-to-one and onto. Thus, its inverse is unique, concluding the demonstration.

\section{REFERENCES}

[1] P. P. Vaidyanathan, Multirate Systems and Filter Banks. Englewood Cliffs, NJ: Prentice-Hall, 1993.

[2] H. S. Malvar, Signal Processing with Lapped Transforms. Norwood, MA: Artech House, 1992.

[3] M. Vetterli and D. Le Gall, "Perfect reconstruction filter banks: Some properties and factorizations," IEEE Trans. Acoust., Speech, Signal Processing, vol. 37, pp. 1057-1071, July 1989.

[4] J. W. Woods, Ed., Subband Coding of Images. Hingham, MA: Kluwer, 1991.

[5] C. Diab, R. Prost, and R. Goutte, "Error-free image decomposition/reconstruction for subband coding schemes," Signal Processing: Image Communication, vol. 2, pp. 53-68, May 1990.

[6] G. Karlsson and M. Vetterli, "Extension of finite length signals for subband coding," Signal Processing, vol. 17, pp. 161-168, 1989.

[7] M. J. Smith and S. L. Eddins, "Analysis/synthesis techniques for subband image coding," IEEE Trans. Acoust., Speech, Signal Processing, vol. 38, pp. 1446-1456, Aug. 1990. 
[8] S. Martucci, "Signal extension and noncausal filtering for subband coding of images," in SPIE VCIP '91: Visual Communication, vol. 1605, Nov, 1991, pp. 137-148.

[9] H. Kiwa, K. Nishikawa, and M. Iwahashi, "A development of symmetric extension method for subband image coding," IEEE Trans. Image Processing, vol. 3, pp. 78-81, Jan. 1994.

[10] R. H. Bamberger, S. L. Eddins, and V. Nuri, "Generalized symmetric extensions for size-limited multirate filter banks," IEEE Trans. Image Processing, vol. 3, pp. 82-87, Jan. 1994.

[11] R. L. de Queiroz, "Subband processing of finite length signals without border distortions," in Proc. Int. Conf. Acoust., Speech, Signal Processing, vol. IV, 1992, pp. 613-616.

[12] J. N. Bradley and V. Faber, "Perfect reconstruction with critically sampled filter banks and linear boundary conditions," preprint.

[13] V. Nuri and R. H. Bamberger, "A theorectical framework for the analysis and design of size-limited multirate filter banks," Proc. Int. Symp. Time-Frequency Time-Scale Anal., Victoria, Canada, Oct. 1992.

[14] R. L. de Queiroz and K. R. Rao, "Time-varying lapped transforms and wavelet packets," IEEE Trans. Signal Processing, vol. 41, pp. 3293-3305, Dec. 1993.

[15] C. Herley, J. Kovacevic, K. Ramchandran, and M. Vetterli, "Tilings of the time-frequency plane: Construction of arbitrary orthogonal bases and fast tiling algorithms," IEEE Trans. Signal Processing, vol. 41, pp. 3341-3359, Dec. 1993

\section{Blind Restoration of Linearly Degraded Discrete Signals by Gibbs Sampling}

\author{
Rong Chen and Ta-Hsin $\mathrm{Li}$
}

\begin{abstract}
This paper addresses the problem of simultaneous parameter estimation and restoration of discrete-valued signals that are blurred by an unknown FIR filter and contaminated by additive Gaussian white noise with unknown variance. Assuming that the signals are stationary Markov chains with known state space but unknown initial and transition probabilities, Bayesian inference of all unknown quantities is made from the blurred and noisy observations. A Monte Carlo procedure, called the Gibbs sampler, is employed to calculate the Bayesian estimates. Simulation results are presented to demonstrate the effectiveness of the method.
\end{abstract}

\section{INTRODUCTION}

Suppose a discrete-valued (digital) signal $\left\{x_{t}\right\}$ is blurred by an FIR linear filter $\left\{\phi_{i}\right\}$ and contaminated by additive noise $\left\{\epsilon_{t}\right\}$, so that the observed signal $\left\{y_{t}\right\}$ can be written as

$$
y_{t}=\sum_{i=0}^{q} \phi_{i} x_{t-i}+\epsilon_{t} \quad(t=1, \cdots, n) .
$$

The so-called blind restoration problem is to simultaneously estimate the filter $\left\{\phi_{i}\right\}$ and to recover the signal $\left\{x_{t}\right\}$ solely from the observed data record $\left\{y_{t}\right\}$ along with some partial statistical information about $\left\{x_{t}\right\}$. This problem stems from the equalization of digital communication channels in which the signals take only discrete values (e.g., [1], [14]).

In the absence of noise, the restoration (or deconvolution) problem can be approached in many different ways under the assumption

Manuscript received April 3, 1993; revised March 17, 1995. The associate editor coordinating the review of this paper and approving it for publication was Dr. Barry Sullivan.

The authors are with the Department of Statistics, Texas A\&M University, College Station, TX 77843-3143 USA.

IEEE Log Number 9413859. that the $x_{t}$ are independent and identically distributed (i.i.d.) (e.g., [1], [4], [7]). In particular, an efficient method along the lines of inverse filtering has been proposed ([9]-[11]) that explicitly utilizes the discreteness of $\left\{x_{t}\right\}$ yet does not require the stationarity or other statistical information of $\left\{x_{t}\right\}$.

In this correspondence, we deal with the blind restoration problem under a Bayesian framework and by Gibbs sampling. The Gibbs sampling has been successfully applied to the ordinary image restoration problem by Geman and Geman [6] under the assumption that the filter $\left\{\phi_{i}\right\}$ and the statistical parameters of $\left\{x_{t}\right\}$ and $\left\{\epsilon_{t}\right\}$ are all available. In the present correspondence, we include these parameters in the list of unknowns and estimate them simultaneously with the signal $\left\{x_{\ell}\right\}$.

\section{Formulation of THE PROBlem}

Assume that the signal $\left\{x_{t}\right\}$ in (1) is a stationary first-order Markov chain with known state space $\mathcal{A}:=\left\{a_{1}, \cdots, a_{k}\right\}$ but unknown initial probabilities $\theta_{i}:=\operatorname{pr}\left(x_{1-q}=a_{i}\right)$ and unknown transition probabilities $\theta_{i j}:=\operatorname{pr}\left(x_{t}=a_{j} \mid x_{t-1}=a_{i}\right)$. It is clear that the probabilities should satisfy the constraints $\sum_{i=1}^{k} \theta_{i}=1$ and $\sum_{j=1}^{k} \theta_{i j}=1$ for $i=1, \cdots . k$. Let $\theta$ denote the collection of these probabilities, namely $\theta:=\left\{\theta_{i}, \theta_{i j}: i, j=1 . \cdots, k\right\}$. Although extensions to higher order Markov chains are quite straightforward, we restrict our effort to the first-order case for the simplicity of presentation. Assume further that $\left\{\epsilon_{t}\right\}$ in (1) is Gaussian white noise with zero-mean and unknown variance $\sigma^{2}$ and is independent of $\left\{x_{t}\right\}$.

Under these assumptions, the main objective of this correspondence is to simultaneously reconstruct the signal $\boldsymbol{x}:=\left\{x_{1-q}, \cdots, x_{n}\right\}$ and estimate the FIR filter $\phi:=\left[\phi_{0}, \cdots, o_{q}\right]^{\prime}$ along with the statistical parameters $\sigma^{2}$ and $\boldsymbol{\theta}$ on the basis of the data record $\boldsymbol{y}:=$ $\left\{y_{1}, \cdots, y_{n}\right\}$. Note that the values $x_{1-q}, \cdots, x_{0}$ (that are outside the observation interval) are also included in $x$ for reconstruction and that the filter $\phi$ can be minimum phase or nonminimum phase. Noncausal FIR filters can be accommodated into the problem by a transformation of time index.

\section{BAYESIAN APPROACH}

The problem is solved under a Bayesian framework: First, the unknown quantities $\boldsymbol{x}, \boldsymbol{\phi}, \sigma^{2}$, and $\boldsymbol{\theta}$ are regarded as realizations of random variables with suitable prior distributions. The Gibbs sampler, a Monte Carlo method, is then employed to calculate the minimum mean-squared error (MMSE) estimates and/or the maximum a posteriori (MAP) estimates of the unknowns.

\section{A. Prior Distributions}

In principle, prior distributions are used to incorporate our knowledge of the parameters, and less restrictive (or less informative) priors should be employed when such knowledge is limited. Computational complexity is another consideration that affects the selection. Conjugate priors are usually used to obtain simple analytical forms for the resulting posterior distributions (e.g., [2]). To make the Gibbs sampler more computationally efficient, the priors should also be chosen such that the conditional posterior distributions, as we shall see next, are easy to simulate.

For the restoration problem described above, the following priors are used in our procedure: to the filter $\phi$, we impose a Gaussian distribution $p(\phi) \sim N\left(\phi_{0} . \Sigma_{0}\right)$, and to the noise variance $\sigma^{2}$ we impose an inverted chi-square distribution $p\left(\sigma^{2}\right) \sim \lambda^{-2}(\nu ; \lambda)$, i.e., $\nu \lambda / \sigma^{2} \sim \lambda^{2}(\nu)$. Note that large values of $\Sigma_{0}$ and small values of $\nu$ and $\lambda$ correspond to less informative priors. Further, we use independent Dirichlet distributions as priors of $\theta_{i}$ and $\theta_{i j}$. 